\title{
Primary mediastinal seminoma
}

\author{
Aleksandra Napieralska, Wojciech Majewski, Wojciech Osewski, Leszek Miszczyk \\ Radiotherapy Department, Maria Skłodowska-Curie Memorial Cancer Center and Institute of Oncology, Gliwice Branch, Gliwice, Poland \\ Contributions: (I) Conception and design: A Napieralska; (II) Administrative support: W Majewski, L Miszczyk; (III) Provision of study materials or \\ patients: A Napieralska; (IV) Collection and assembly of data: A Napieralska, W Osewski; (V) Data analysis and interpretation: A Napieralska, L \\ Miszczyk, W Majewski; (VI) Manuscript writing: All authors; (VII) Final approval of manuscript: All authors. \\ Correspondence to: Aleksandra Napieralska. Radiotherapy Department, Maria Skłodowska-Curie Memorial Cancer Center and Institute of Oncology, \\ Gliwice Branch, Ul. Wybrzeże AK 15, Gliwice 44-101, Poland. Email: olanapieralska@gmail.com.
}

Background: The objective of this study was to evaluate the long term treatment results of patients with primary mediastinal seminoma.

Methods: Sixteen patients aged 21-46 diagnosed with primary mediastinal seminoma between 1983 and 2014. Mean size of the tumor was $65 \mathrm{~cm}^{2}$. In all patients gonadal involvement was excluded. In 6 patients, metastases to regional lymph nodes were found and none of them presented with distant metastases. HCG was elevated in 6 patients (38\%). Eight patients underwent surgery as first-line of the treatment: 7 partial and 1 complete resection of the tumor. Chemotherapy (CTH) and radiotherapy (RTH) were methods of treatment in 14 cases. Tumor was irradiated to total dose of 36.0-50.4 (median 42.5) Gy. In statistical analysis overall survival (OS) was calculated using Kaplan-Meier method.

Results: During median time of 11 years of the follow-up, complete or partial regression (PR) of the tumor was seen in all patients after primary treatment. Recurrence of the tumor was seen in 3 patients. The 5-, 10- and 15-year local control rates were 75\%. One was treated with CTH, other two with RTH. All of them responded with complete regression (CR) of the tumor. Three patients died during the follow-up. All others are alive without disease. The 5-, 10- and 15-year OS was 100\%, 91\% and 91\% respectively.

Conclusions: Chemoradiotherapy of primary mediastinal seminoma gives satisfactory treatment results with good local control rate. The treatment outcome is comparable to primary testicular seminoma.

Keywords: Seminoma; chemotherapy (CTH); germ cell tumors; radiotherapy (RTH); surgery; mediastinal seminoma

Submitted May 09, 2018. Accepted for publication Jun 18, 2018.

doi: $10.21037 /$ jtd.2018.06.120

View this article at: http://dx.doi.org/10.21037/jtd.2018.06.120

\section{Introduction}

Mediastinal seminomatous germ cell tumors are rare neoplasm first reported by Woolner et al. in 1955 (1). Germ cell tumors account for $1-4 \%$ of mediastinal tumors and seminomas are less frequent than non-seminomatous tumors (2-4). Two most common extragonadal sites are the mediastinum and retroperitoneum $(3,4)$. Due to the slow growth of seminomas, most of them have been found large and bulky at the time of a diagnosis. Symptoms are not characteristic and there are patients in whom this type of tumor is diagnosed incidentally $(2,3,5-8)$.
Treatment strategies differ among studies; however, since the introduction of chemotherapy (CTH) into the treatment of those patients, it has become the main and the first-line treatment modality (4-6,9-11). The role of radiotherapy (RTH) or surgery as post CTH treatment has not yet been established (11). Reported 5- and 10 -year overall survival (OS) of patients with primary mediastinal seminomas ranged from $87 \%$ to $100 \%$ and from $75 \%$ to $100 \%$, respectively, and is similar to testicular seminoma treatment results described in the literature (2-5,9-13). 


\section{Aim of the study}

The aim of our study was to assess the long term results of treatment of patients with primary mediastinal seminoma in a single institution.

\section{Methods}

A retrospective study of patients with primary mediastinal seminoma treated in our Institution between 1983 and 2014 was performed. Among patients diagnosed with seminoma, we found 16 patients with primary mediastinal seminoma. All the patients were men and their age at the time of the diagnosis ranged from 21 to 46 years, with a mean of 33 (median 34).

The study was performed according to the Helsinki Declaration and the Institutional Review Board Committee.

In all the cases, the diagnosis was based on diagnostic imaging and pathologic examination of the tumor tissue samples obtained during biopsy or surgery. In 14 cases, tumor was found on a computed tomography (CT) of the chest and in 2 cases (treated in the earlier years of a study) on the chest $\mathrm{X}$-ray. In 9 cases, tumor was constricting nearby organs and in 4 cases, it was infiltrating surrounding tissues. Mean tumor area was $65 \mathrm{~cm}^{2}$ and the tumors ranged from 4.5 to $17.7 \mathrm{~cm}$ in the greatest dimension. In six patients, metastases to regional lymph nodes were found. None of the patients presented with distant metastases. According to International Germ Cell Cancer Collaborative Group classification, all the patients were in good prognosis group (14).

Evaluation of pretreatment $\beta$-HCG level was documented in 8 cases and ranged from 0.00 to $452 \mathrm{U} / \mathrm{mL}$. In the above mentioned cases, $\beta$-HCG was elevated in 6 patients $(38 \%$ of all patients). All but one patient were in a good performance status at the time of the diagnosis (ECOG 0-1). One patient was in a poor performance status (ECOG 3) due to severe symptoms of superior vena cava syndrome. At the same time, a few days after the start of RTH, his symptoms decreased and he remained in a good performance status hereinafter. In all the cases, physical examination of the testis and ultrasound were performed to exclude gonadal involvement. All of the patients had abdominal CT or ultrasound performed prior to the commencement of the treatment and no abnormalities were found. None of the patients had a previous history of testicular neoplasms or other types of cancer.

One patient had been diagnosed with the tumor incidentally. The rest of patients developed symptoms for a median time of 4 months (range, $0-12$ months). The most common symptoms were: chest pain (44\%), dyspnea (38\%) and cough (38\%) The others were: fever (25\%), superior vena cava obstruction (19\%), swelling of face or neck (19\%) weight loss $(13 \%)$ and palpable tumor in a supraclavicular fossa $(13 \%)$.

In all the cases, primary mediastinal seminoma was treated with radical intention. Eight patients underwent surgery as the first-line treatment, however only in one case a complete removal of the tumor was performed and other patients had partial resection. CTH was a method of a treatment in 14 patients, in 8 of them as the first-line treatment. All of them received 2 to 6 cycles (median 5) of BEP (bleomycin 30 j.m. intravenous days 1,8 and 16 , etoposide $100 \mathrm{mg} / \mathrm{m}^{2}$ intravenous days $1-5$, cisplatin $20 \mathrm{mg} / \mathrm{m}^{2}$ intravenous days $1-5$ in 21-day cycles). After the CTH, the size of the tumor was assessed based on a comparative radiologic analysis-images taken before and after the treatment were compared for all the cases.

Tumor response was classified, as follows: complete regression (CR) was defined as complete disappearance of all clinical, radiologic and biochemical ( $\beta-\mathrm{HCG}$ ) evidence of the disease. Partial regression (PR) was defined as a decrease of tumor size in clinical or radiologic evaluation of the disease with decrease of $\beta$-HCG level after the treatment. Progression of the disease was defined as increase in lesion size, occurrence of new lesions or elevation of tumor marker.

After CTH, the progression of the tumor (i.e., the increase in lesion size) was found in one patient, one responded with CR and the others with PR of the tumor.

RTH was a method of treatment in 14 cases. In 11 patients, RTH followed CTH, whereas, in one case, it was conducted after CTH and surgery. Additionally, in 2 cases, it was conducted without CTH after partial resection of the tumor. After RTH, four patients responded with PR and eight with CR of the tumor mass. The characteristics of the treatment methods used in particular patients are presented in Table 1.

In the majority of the cases, conformal technique of RTH (two or three fields' technique) was used. Intensity modulated radiation therapy (IMRT) was performed in 3 cases. Irradiated fields included a tumor or a tumor bed with mediastinum and, in 3 cases, also supraclavicular regions. In 3 cases, boost of 9-14.4 Gy was delivered to the tumor. In all the patients irradiated after 2002, treatment was planned based on CT images. In 3 patients, PET- 
Table 1 Characteristics of treatment methods in particular patients

\begin{tabular}{lcccccccc}
\hline Patient & Surgery & CTH & Number of cycles & Result of CTH & RTH & TD/FD (Gy) & Result of RTH & Recurrence \\
\hline 1 & Yes, NR & Yes & 4 & PR & Yes & $45.0 / 1.8$ & PR & No \\
2 & No & Yes & 6 & N/D & Yes & N/D & CR & No \\
3 & Yes, NR & Yes & N/D & N/D & Yes & N/D & PR & No \\
4 & No & Yes & N/D & N/D & Yes & N/D & CR & No \\
5 & No & Yes & N/D & N/D & Yes & N/D & CR & No \\
6 & No & Yes & 6 & CR & Yes & $39.6 / 1.8$ & CR & No \\
7 & Yes, NR & No & - & - & Yes & $40.0 / 2.0$ & CR & No \\
8 & Yes, NR & No & - & - & Yes & $40.0 / 2.0$ & CR & Yes \\
9 & No & Yes & 4 & PR & Yes & $45.0 / 1.8$ & N/D & No \\
10 & Yes, NR & Yes & 4 & PR & Yes & $36.0 / 1.8$ & N/D & No \\
11 & No & Yes & 6 & PR & Yes & $36.0 / 1.8$ & CR & No \\
12 & Yes, NR & Yes & 3 & PR & No & - & - & Yes \\
13 & No & Yes & 6 & PR & Yes & $50.0 / 2.0$ & CR & Yes \\
14 & No & Yes & 6 & PR & Yes & $50.0 / 2.0$ & PR & No \\
15 & Yes, R & Yes & 4 & PR & Yes & $50.4 / 1.8$ & PR & No \\
16 & Yes, NR & Yes & 2 & CR & No & - & - & No \\
\hline
\end{tabular}

CR, complete regression; CTH, chemotherapy; FD, fraction dose; N/D, not described; NR, non-radical; PR, partial regression; R, radical; $\mathrm{RTH}$, radiotherapy; TD, total dose.

CT was used to improve treatment planning. Treated regions were irradiated with the total dose of 36.0-50.4 (median 42.5) Gy with fraction dose of 1.8-2.0 Gy. In these cases that were treated after the year of 2003 , retrospective analysis of treatment plans was performed (it was impossible to retrieve earlier plans due to the changes in the treatment planning system). In one case, RTH was delivered using ${ }^{60} \mathrm{Co}$ photons, whereas in all others, with the use of 6-23 MV X photons beams.

Clinical target volume (CTV) consisted of a gross tumor volume (GTV) (13 cases) or tumor bed (1 case) with mediastinum (9 cases). In 3 patients, supraclavicular region was part of the CTV. Planned target volumes (PTVs) were created by adding additional margins to CTV in order to correct for inaccuracies in the delivery system (setup margins) or interfraction and intrafraction of organ motions. Mean volumes of CTV and PTV were $405 \pm 268.3$ and $480 \pm 451.8 \mathrm{~cm}^{2}$, respectively. CTV and PTV boost consisted of GTV with margins only. Mean volumes of CTV and PTV boost were $296 \pm 104.7$ and $277 \pm 343.3 \mathrm{~cm}^{2}$, respectively. Mean dose delivered to the heart ranged from 0 to 21 (median 12) Gy and 20\% volume $\left(\mathrm{V}_{20}\right)$ of the heart received from 0 to 43 (median 23) Gy. Mean dose delivered to the lungs varied from 0 to 16 (mean 9) Gy and $20 \%$ volume $\left(\mathrm{V}_{20}\right)$ of lungs received from 0 to 30 (mean 17) Gy.

After the chemoradiotherapy one patient had surgical resection of the remaining tumor mass. On a pathological evaluation of resected tissues tumor cells were not found.

All patients were observed for a median time of 11 years. In a statistical analysis, OS and relapse-free survival was calculated using Kaplan-Meier method. Various patients' characteristics such as age, ECOG performance status, the presence of symptoms, $\beta$-HCG concentration, tumor infiltration of surrounding tissues, the presence of nodal metastases and treatment factors as year of start treatment and therapeutic modalities were included in the univariate analysis to identify their impact on OS. Comparisons were made with the use of the log-rank test. A $P$ value of $\leq 0.05$ was regarded as being statistically significant. Follow-up time was defined from the date of the diagnosis to the date of death due to any reason or the last contact. 


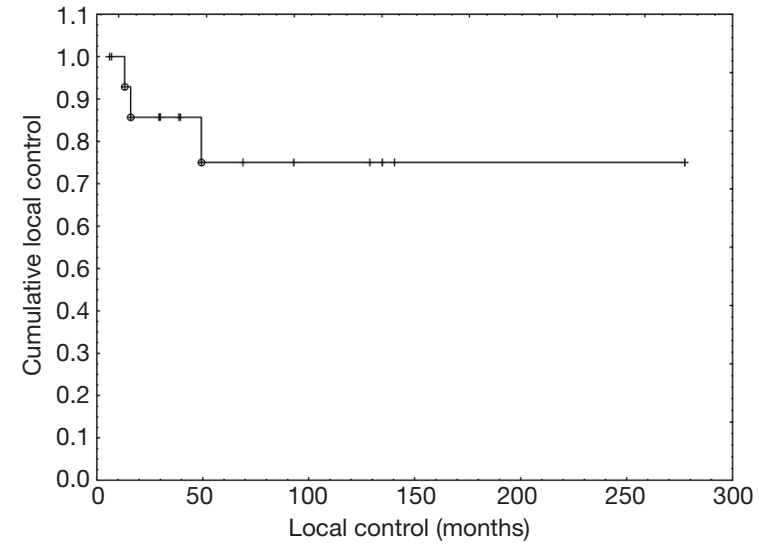

Figure 1 Local control rate.

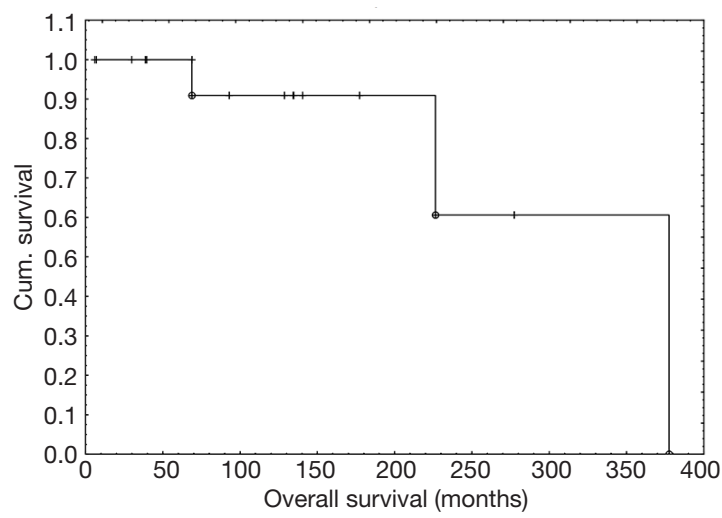

Figure 2 Overall survival of all patients.

\section{Results}

Follow-up ranged from 5 to 378 months (median 11 years). Complete or PR of the tumor was observed in all the patients after the treatment. Recurrence was found in 3 patients during the follow-up after 13 to 49 months following the primary treatment. In one case, a salvage CTH based on 5 cycles of BEP (patient did not received CTH during the primary treatment) resulted in a CR of the tumor. Two other patients received RTH. In one of them, recurrent tumor was located in supraclavicular fossa and has been irradiated with the total dose of 23 Gy (this patient had been previously irradiated on mediastinal and supraclavicular fields with the total dose of $50 \mathrm{~Gy}$ ). The second one, who had previously not received RTH, was irradiated on mediastinal and supraclavicular regions with the total dose of 34 Gy. Both of them responded with a CR of a tumor mass. In one of them, 30 years after the primary treatment, a new seminomatous tumor in the retroperitoneal region was found. The 5-, 10- and 15-year relapse-free survival rates were $75 \%$ (Figure 1).

None of the patients developed metastases to distant organs during the follow-up. No secondary neoplasms or testicular tumors were seen during the follow-up. After the treatment, CT imaging revealed radiological fibrosis of the irradiated lung volume in 8 patients $(50 \%)$, however, without signs of any clinical symptoms. Cardiac/circulatory diseases were diagnosed in 5 patients (31\%). Three of them were diagnosed with hypertension, one with myocarditis and one suffered from ischemic heart disease, arrhythmia and cardiogenic shock.

During the follow-up, 3 patients (19\%) died. All others are still alive and free of the disease. The 5-, 10- and 15 -year OS was $100 \%, 91 \%$ and $91 \%$, respectively (Figure 2). Age over 40 years was the only one independent factor that influenced OS (see Table 2, Figure 3).

\section{Discussion}

Mediastinal germ cell tumors are uncommon and seminomas are less frequent than non-seminomas (2-4). Despite many differences between this two groups of tumors (such as treatment approach, OS or risk of metastasis) most of the authors reported treatment outcomes for all of them together. As the number of seminomas is usually low, it is difficult to draw definite conclusions from such combined studies.

Primary mediastinal seminomas predominantly occur in young males; however reports concerning female patients with seminomatous tumors have been published $(2,6)$. In the present study, median patient age at diagnosis was 33 years which is in agreement with other reports $(2,4)$.

According to other authors, approximately $20 \%$ to $40 \%$ of patients are asymptomatic at the time of diagnosis $(2,3,5)$. Our research shows that only 1 patient (6\%) was diagnosed incidentally and all others developed symptoms for median time of 4 months. Superior vena cava syndrome was present in $19 \%$ of our patients and other authors described the incidence of $6 \%$ to $30 \%$ (2-4). The tumors size ranged from 4.5 to $17.7 \mathrm{~cm}$ in the greatest dimension which suggest that they had been growing for quite a long time before patients developed symptoms. $\beta$-HCG level was elevated in $38 \%$ of patients. Other authors reported elevation of $\beta-\mathrm{HCG}$ in 0 to $43 \%$ in primary mediastinal seminoma patients $(2,4-6,12,14)$. At the same time, in patients with primary testicular seminoma, the elevation of $\beta-\mathrm{HCG}$ is observed 
Table 2 Univariate analysis of patients and treatment characteristics for their influence on overall survival

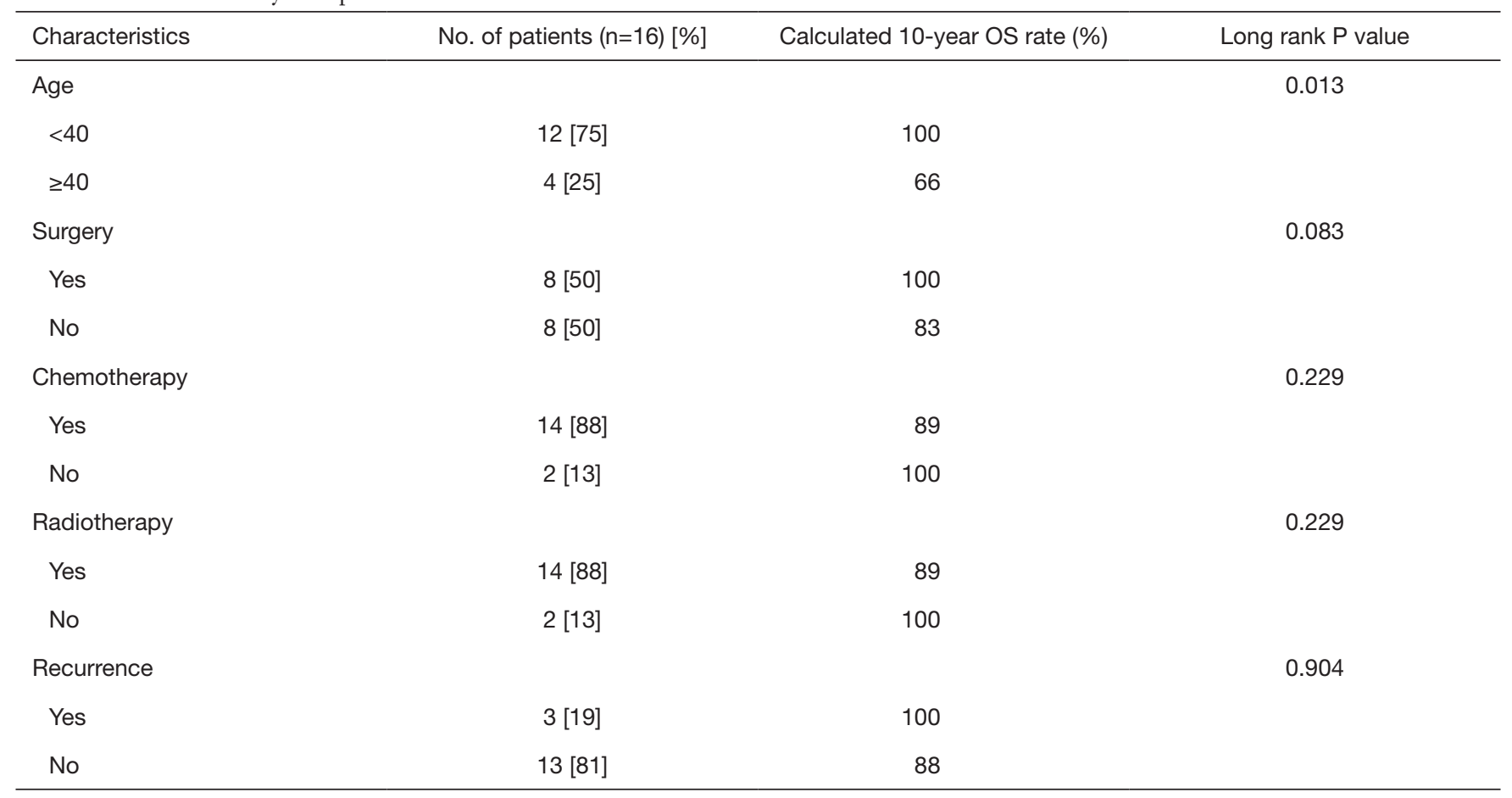

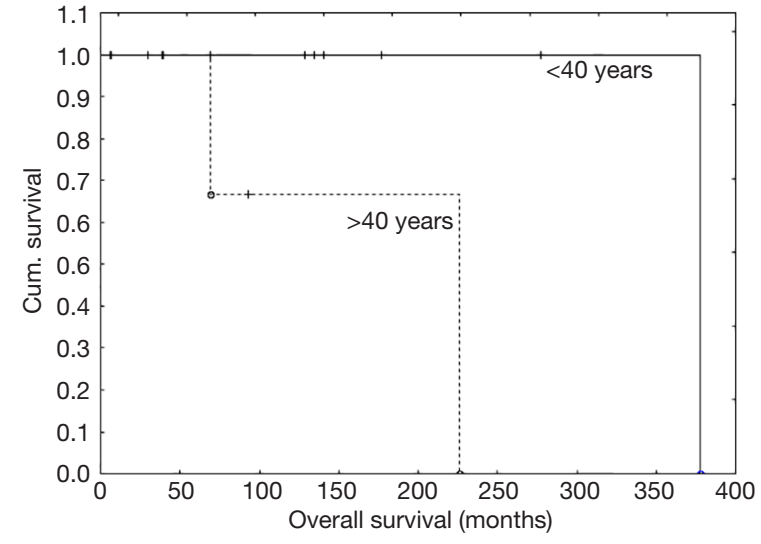

Figure 3 Overall survival in patients over or under 40 years old $(\mathrm{P}=0.013)$.

in $7.5-19 \%$ of patients (15-17). A relatively high number of primary mediastinal seminoma patients with elevated $\beta$-HCG might be associated with large volume of these tumors. A weak point of the aforementioned assumption is the proportion of patients who had a pre-treatment $\beta$-HCG level being assessed. In our study, the above was caused by the fact that in half of patients the primary treatment was surgery performed outside our center. Besides, in many other research, the rate of $\beta-H C G$ assessment was similar to ours and ranged from $36 \%$ to $90 \%(3,5,11,15,18)$.

Hartmann et al. reported the occurrence of testicular cancers during long term follow-up of patients with primary mediastinal seminoma. The risk of developing metachronous testicular cancer was statistically increased. The cumulative 10-year risk of developing testicular cancer after a diagnosis of extragonadal germ cell tumor was $10.3 \%$ although patients with seminomatous histology and mediastinal location of primary tumor were characterized by the lower risk of $1.4 \%$ and $6.2 \%$, respectively (19). In our study, during the follow-up, none of the patients developed neoplasm of the testis (but one of them was diagnosed with large seminomatous tumor in the retroperitoneal region 30 years after primary treatment). This might be associated with a relatively low number of patients in our group or could be a result of a significantly decreased risk of developing metachronous testicular cancer. Hartmann's et al. analysis covers the period between 1975 and 1996 when CTH was not standard treatment method for patients with mediastinal germ cell tumors. Patients with testicular cancers treated with CTH were found to have lower risk of developing metachronous testicular cancer than those treated without CTH $(20,21)$. Therefore, the application of 
CTH in patients with extragonadal germ cell tumors might have a similar influence on risk of developing testicular cancers during follow-up but this hypothesis must be further verified in the future.

Moran et al. reported that age over 37 years old is associated with a worsened prognosis and we found similar association in our group (3). Patients older than 40 years had only $66 \% 10$-year OS compared to $100 \%$ in younger patients $(\mathrm{P}=0.013)$. No other significant factors associated with OS were noted (see Table 2).

The 5 -year progression free survival reported by other authors and IGCCCG ranged from $82 \%$ to $88 \%$ $(4,11,12,14)$. The reported 5 - and 10 -year OS rates ranged from $67 \%$ to $100 \%$ and from $75 \%$ to $100 \%$, respectively (2-6,9-12). These patients' outcomes are similar with our observations. Treatment modalities differ among studies, however since the introduction of CTH into the treatment of mediastinal germ cell tumors, it has become the main treatment modality $(4-6,10,11)$. What is more, it seems that CTH is more efficient than RTH $(2,4,6,12,14)$. The role of RTH or surgery as post-CTH treatment has not yet been established $(2,11,18)$. In the majority of the recent reports patients received only $\mathrm{CTH}$ as main treatment modality with part of the patients treated with adjuvant RTH or resection of post-CTH residual tumor mass (3,4,8-11,21). Takeda et al. reported treatment outcomes of 13 patients with mediastinal seminomas among whom $77 \%$ of patients had radical resection of the tumor and RTH and CTH were used in $85 \%$ and $46 \%$ of patients, respectively. Despite the radical surgery and postoperative treatment, 5-year OS was only $75 \%$ and 1 patient died during the surgery and one developed a severe complication (life-threatening hypoxemia) after resection (2). Osada et al. described treatment outcomes of chemoradiotherapy alone with 5- and 10-year OS of 100\% (5). In study of Liu et al., the use of RTH was associated with a statistically significant improvement in OS (10).

In a study of Rivera $e t$ al., concerning the surgery in patients with primary mediastinal germ cell tumors, a resection of the tumor was independent prognostic factor for significantly better OS, but most of the patients in this group presented with non-seminomatous tumors (68\%) (18). Patients who underwent surgery in our study had better OS, although the difference was not statistically significant $(\mathrm{P}=0.08369)$ and most of them had only partial resection of the tumor.

One patient had surgical removal of residual tumor after primary chemoradiotherapy and tumor cells were not found in pathologic evaluation of resected tissues. Bokemeyer $e t a l$. and Takeda $e t a l$. reported that resection of post-treatment residual mass demonstrated high probability for necrosis which is similar with our observation $(2,4,11)$. The application of fluorodeoxyglucose-positron emission tomography/CT (FDG-PET-CT) in the prediction of viable tumor in post-CTH testicular seminoma patients and in the evaluation of treatment results had been reported $(11,22)$.

FDG-PET-CT was performed in 9 cases (56\%) and only in one case a high uptake of FDG in mediastinal postCTH mass was revealed. None of the patients who received radiochemotherapy had high uptake of FDG in PET-CT after combined treatment.

The 5- and 10-year OS for patients with testicular and mediastinal seminomas of $92 \%$ and $86 \%$, and $100 \%$ and $91 \%$, reported by our Institution, support the conclusion that no distinction has to be made between those entities in a modern multidisciplinary approach $(4,13)$.

\section{Conclusions}

$\mathrm{CTH}$ as a first line of treatment is recommended in patients with primary mediastinal seminoma. The OS in this group of patients is good and comparable with patients with primary testicular seminomas.

\section{Acknowledgements}

None.

\section{Footnote}

Conflicts of Interest: The authors have no conflicts of interest to declare.

Ethical Statement: The study was approved by the Institutional Review Board Committee.

\section{References}

1. Woolner LB, Jamplis RW, Kirklin JW. Seminoma (germinoma) apparently primary in the anterior mediastinum. N Engl J Med 1955;252:653-7.

2. Takeda S, Miyoshi S, Ohta M, et al. Primary germ cell tumors in the mediastinum: a 50-year experience at a single Japanese institution. Cancer 2003;97:367-76.

3. Moran CA, Suster S, Przygodzki RM, et al. Primary germ cell tumors of the mediastinum: II. Mediastinal seminomas 
-- a clinicopathologic and immunohistochemical study of 120 cases. Cancer 1997;80:691-8.

4. Bokemeyer C, Nichols CR, Droz JP, et al. Extragonadal germ cell tumors of the mediastinum and retroperitoneum: results from an international analysis. J Clin Oncol 2002;20:1864-73.

5. Osada H, Kojima K, Yamate N. Primary mediastinal seminoma. Efficacy of chemoradiotherapy alone. Jpn J Thorac Cardiovasc Surg 1998;46:810-4.

6. Giaccone G. Multimodality treatment of malignant germ cell tumours of the mediastinum. Eur J Cancer 1991;27:273-7.

7. Lee H, Lim JK, Lee SY, et al. Granulomatous reaction of primary mediastinal seminoma leading to diagnostic delay: a case report. J Thorac Dis 2018;10:E98-102.

8. Dechaphunkul A, Sakdejayont S, Sathitruangsak C, et al. Clinical Characteristics and Treatment Outcomes of Patients with Primary Mediastinal Germ Cell Tumors:10Years' Experience at a Single Institution with a BleomycinContaining Regimen. Oncol Res Treat 2016;39:688-94.

9. Sudour-Bonnange H, Faure-Conter C, Martelli H, et al. Primary mediastinal and retroperitoneal malignant germ cell tumors in children and adolescents: Results of the TGM95 trial, a study of the French Society of Pediatric Oncology (Société Française des Cancers de l'Enfant). Pediatr Blood Cancer 2017;64(9).

10. Liu TZ, Zhang DS, Liang Y, et al. Treatment strategies and prognostic factors of patients with primary germ cell tumors in the mediastinum. J Cancer Res Clin Oncol 2011;137:1607-12.

11. Sakaguchi $Y$, Isowa N. Successful resection of mediastinal seminoma evaluated the response to induction chemotherapy with fluorodeoxyglucose-positron emission tomography. Ann Thorac Cardiovasc Surg 2012;18:45-7.

12. Wang JL, Yu H, Guo Y, et al. A single institution, retrospective study of treatment experience in primary mediastinal germ cell tumors: elucidating the significance of systemic chemotherapy. Chin Med J 2012;125:626-30.
13. Majewski W, Majewski S, Maciejewski A, et al. Adverse effects after radiotherapy for early stage (I, IIa, IIb) seminoma. Radiother Oncol 2005;76:257-63.

14. International Germ Cell Consensus Classification: a prognostic factor-based staging system for metastatic germ cell cancers. International Germ Cell Cancer Collaborative Group. J Clin Oncol 1997;15:594-603.

15. Liu Y, Wang Z, Peng ZM, et al. Management of the primary malignant mediastinal germ cell tumors: experience with 54 patients. Diagn Pathol 2014;9:33.

16. Hofmockel G, Theiss M, Gruss A. Treatment results and long-term follow-up in stage I seminoma patients. Oncol Rep 1996;3:107-10.

17. Bruns F, Raub M, Schaefer U, et al. No predictive value of beta-hCG in patients with stage I seminoma-results of a long-term follow-up study after adjuvant radiotherapy. Anticancer Res 2005;25:1543-6.

18. Rivera C, Arame A, Jougon J, et al. Prognostic factors in patients with primary mediastinal germ cell tumors, a surgical multicenter retrospective study. Interact Cardiovasc Thorac Surg 2010;11:585-9.

19. Hartmann JT, Fossa SD, Nichols CR, et al. Incidence of metachronous testicular cancer in patients with extragonadal germ cell tumors. J Natl Cancer Inst 2001;93:1733-8.

20. Andreassen KE, Grotmol T, Cvancarova MS, et al. Risk of metachronous contralateral testicular germ cell tumors: a population-based study of 7,102 Norwegian patients (1953-2007). Int J Cancer 2011;129:2867-74.

21. Schaapveld M, van den Belt-Dusebout AW, Gietema JA, et al. Risk and prognostic significance of metachronous contralateral testicular germ cell tumours. Br J Cancer 2012;107:1637-43.

22. Becherer A, De Santis M, Karanikas G, et al. FDG PET is superior to CT in the prediction of viable tumour in post-chemotherapy seminoma residuals. Eur J Radiol 2005;54:284-8.
Cite this article as: Napieralska A, Majewski W, Osewski W, Miszczyk L. Primary mediastinal seminoma. J Thorac Dis 2018;10(7):4335-4341. doi: 10.21037/jtd.2018.06.120 